\title{
Translating texts into care: classification issues raised by evidence-based practice in the UK health sector
}

\author{
Elisabeth Davenport \\ Napier University \\ Edinburgh, Scotland
}

\begin{abstract}
The problem of translating texts into care (where practitioners are mandated to base their work on the literary corpus) has been construed in several ways: in terms of time management, in terms of physical access, and in terms of adequate surrogates for texts (translations) like indexes, abstracts, systematic reviews. In some cases, there may be a deeper problem of incommensurability. The nursing profession in the UK, a newly professionalized group faced with government mandates to base their practice on medical evidence, may constitute such a case. EBM (evidence-based medicine, or 'text') poses challenges for nurses (proponents of 'caritas'). This paper reviews epistemological and ontological problems identified in previous research on evidence-based nursing practice, which suggests that incommensurability of the medical and nursing domains is an issue. An additional surrogate for the medical corpus, the clinical guideline, is discussed. When based on inclusive consultation, this may prove to be a hospitable 'translation artefact' for groups whose domains are in conflict. Drawing on theoretical work on 'translation' by Bowker and his colleagues, by Berkenkotter and by Latour, the author explores the status of the clinical guideline as a translation artefact: it is a novel hybrid, which links retrieval, classification and action by combining different warrants.
\end{abstract}

\section{Introduction}

The problem of translating texts into practice was succinctly described by de Solla Price:

In addition to...clinical research, there is supposedly another, perhaps even more numerous variety whose function is that it must be read and evaluated by practitioners who are guided and influenced thereby in their clinical practice. Through we have little direct or indirect evidence of this process, one can see that such use, turning knowledge directly into action must have special library problems. In particular, since the scientific and medical community is not giving rise to further literature in the course of turning clinical research papers into clinical practice, we do not have a process of validating and evaluation (sic) such research by its affected community. The heavy responsibility for proper reading and evaluating such clinical literature is a vital technique that must be learned. (Price, 1981, p. 8) 
The 'heavy responsibility' of closing what may be called 'Price's loop' has been taken up in a number of ways. Foremost is the work of a dedicated cadre who write systematic reviews (McKibbon et al., 1995; Boynton et al., 1997), notably contributors to the Cochrane Collaboration (inspired by Cochrane's plea Cochrane 1972) to consolidate the evidence on which care is based). By synthesizing and evaluating evidence from clusters of randomized clinical trials, systematic reviews can support recommendations for practice that are based on evidence. The position of systematic reviews in the medical corpus is as yet ambiguous as there are inconsistencies in the ways that major indexes accommodate them (Krabshuis, 1998), making retrieval by practitioners less than optimal. Other approaches to turning knowledge into action are beset with problems. Coding, for example, even where codes are designed by practitioners (like the READ codes in the UK), can be inflexible (Williams, 1996) and there are incompatibilities across systems (Cimino, 1995; Henry, 1995). Some codes are not accounted for in UMLS, the 'universal' index, and though work is being done on computer translation across coding schemes (Rector et al., 1995) these are not yet standard components of clinical systems. And there are inconsistencies in the indexing of the major databases. Attempts to make the medical corpus more searchable by busy practitioners can result in lost information unless an experienced searcher familiar with the limitations of different coding and indexing systems, undertakes the retrieval task (Krabshuis, 1997).

In addition to reading and evaluating the literature, representing it in terms which may be appropriated in local practice is a further responsibility. Until recently, local habitats were of little interest to librarians, who aspire to create and maintain universal knowledge representation structures/schemata which transcend particular and ephemeral descriptions. It is easy to understand the "pressure for standardized classification" (Meadows, 1987), but the end result, in the case of medicine, is a complex literary apparatus that can produce arbitrary results in the hands of practitioners constrained by lack of time, supported by different resources, and exhibiting varying degrees of searching skill in their attempts to find material that fits the framework of local needs. Hopes that the intersection of the global and the local (by means of a computer in every ward that can act as an in situ search engine and local archive (Henry, 1995) and combine external material with patient data) cannot be realised where the appropriation problem has not been tackled.

Appropriation is a two-way process, and the problem of capturing local practice and the frameworks that emerge in local practice is an issue in evidence-based medicine. The digital desktop allows representations of the heterogeneous material that constitutes the world of work to be collocated on one screen, and allows details to be captured and observed that that were previously elusive (Star, 1996). Such texts, traces of the world of practice, may make it easier for practitioners to scrutinise and reflect on the acts of local classification that shape their work and to identify areas of practice that are invisible in the literature, though they are capable of representation and worthy of extension and recognition beyond the local habitat. In the world of clinical practice, much of this 'articulation' -- work that gets things done -- (Gerson \& Star, 1986) is undertaken by nurses, and nursing has served as a ready context for the examination of a number of concepts which underly appropriation and must be addressed in any study of evidence based healthcare: legitimacy, authority, recognition, translation. These issues have been addressed in a body of text that emphasizes the importance of domain expertise in categorizing texts and work, and explores the possibilities of convergence between local schemes and those of 
the bibliographic canon. Several of these studies (McCloskey \& Bulechek, 1995; Bowker, Timmermans \& Star, 1995; Bowker \& Star, 1997; Jacob \& Albrechtsen, 1997) analyze the process of legitimation and the nature of the political economy which underlies such legitimation activity in case studies of the Nursing Intervention Classification [NIC].

There are points in common between the process of legitimation that underlies the NIC and the epistemological stake-out that appears to drive the reaction of the nursing profession to evidencebased healthcare in the UK, where the nursing profession has claimed that it is marginalized by mandates to make systematic reviews the basis of healthcare; nursing interventions are not amenable to assessment in terms of the randomized clinical trial, a medical yardstick, and other types of 'evidence' must be considered. This view is summarized in Berragan's (1998) equation of 'the move towards evidence based practice' with 'potential denial of personal ways of knowing such as intuition' (p. 212). The text which follows discusses some problems that arise from claims that nursing knowledge is a distinct domain, and considers the role of clinical guidelines as a translation artefact which may align conflicting points of view and help medical and nursing practitioners to achieve their common objective of improved patient care.

\section{Background: nursing in the UK}

In the UK, the nursing profession is currently challenged by two major developments. The first challenge is a shift from the 'Nightingale model' of apprenticeship in the wards (backed up in the college classroom) to training in the Higher Education sector, either at the level of a first undergraduate degree, or, in the case of experienced practitioners, at Masters level. The process of migration was completed in 1992 (Yeoh, 1995). Recent government initiatives have consolidated the pedagogic shift. Both the Post Registration Education Programme [PREP] and the 'Year 2000' programme will contribute to a level graduate playing field where practitioners continuously update their skills and knowledge base (Urquhart \& Crane, 1995). In addition, evidence based medicine [EBM] will drive fiscal and professional practice in National Health Service [NHS] institutions. Medical, clinical and paramedical staff must base treatment on the results of studies of previous outcomes, not simply on historical precedent or personal bias. (Department of Health, 1991; Department of Health, 1993; Royal College of Nursing, 1996; National Health Service Executive, 1996)

For experienced nursing practitioners who undergo continuing professional training, access to the corpus of evidence may prove problematic, as they (1) may not have access to databases and (2) may not know how to navigate the 'grand structures' offered by major suppliers of data such as Medline, Biosis, and Embase (Farmer and Richardson, 1996). Both of these issues have been raised as inhibitors for practitioners who must work with 'evidence', and, since they are construed as technical problems, training is considered to offer a solution. Underlying these, however, is problem (3): practitioners may find it difficult to reconcile their structural understanding of the canonical textual domain with their understanding of practice (Hicks \& Hennessy, 1995; Hicks et al., 1996; Berragan, 1998). 'Boundary' or 'translation' work is required which can articulate both nurses' perceptions of the component parts of their work, and where these fit into frameworks offered by the medical literature. 


\section{Research approach}

The research topic ('translating texts into care') was explored with the help of a group of experienced nurses acquiring Masters degrees in Queen Margaret College in Edinburgh [QMC]. QMC has trained nurses for many years, before and after the migration to the Higher Education sector, and is talked of in the profession as offering the 'QMC model', which emphasizes understanding of context and quality of life as important factors in effective intervention. Volunteers were sought in the early stages of a Spring semester Research Methods class who would help the author explore evidence-based healthcare as a classification and retrieval problem; but as discussion of this topic developed, it became clear that indexing structures and the role of nursing indexes in the retrieval apparatus were not of primary concern to the practitioners in the class. The recent and ongoing publication of mandatory clinical guidelines was a more pressing challenge, as these were primarily based on randomized clinical trials [RCTs], perceived by many participants as biased by medical ('doctor') input and excluding 'nursing knowledge'. The focus of the project shifted to clinical guidelines, the assumptions and politics that underly them, and nurses' perceptions of their pertinence to nursing practice and the nursing profession. This new path led the researcher to a nexus of activities and actors (represented in textual, electronic and face to face testimony) which constitute a conscientious attempt to span medical and nursing domains in clinical guidelines constructed in an inclusive and transparent way in which the role of the web has been important. Initial analysis of the guideline 'production lattice' (Kling, 1988) has thrown light on a number of classification and retrieval problems faced by practising nurses who engage with the classical medical corpus. It has also clarified the status and role of the clinical guideline as a retrieval and classification tool of a novel sort, a hybrid translation artefact with consequences in the real world. To justify this claim, the text which follows reviews literature on the information habits of nurses, discusses nursing knowledge, and a nursing 'point of view' which may inhibit nurses from translating texts into practice, and concludes with an analysis of clinical guidelines in the Scottish Health Service and their role as novel retrieval and classification tools. The analysis draws on the work of Bowker, Star, Berkenkotter and Latour and their colleagues.

\section{Textual inhibitions}

To support the claim that a clinical guideline can remedy retrieval and classification problems, evidence that problems exist must be supplied: the brief review of the information habits of nurses which follows attempts to do this. In the UK, these issues have been discussed from the standpoint of librarians and information professionals perplexed by lack of engagement, and determined to find a technical solution to the problem (Capel et al.,1997; Bawden \& Robinson, 1997) A much cited review of nursing information habits in 1994 (Urquhart \& Crane, 1994) revealed that one third of nurses did not engage with the literature at all; that one third consulted a few trusted sources not involving searching (trade literature and work of mouth); and that only one third exploited the apparatus of biomedical indexes. The main indexes consulted were SINAHL, and MEDLINE. Bawden \& Robinson (1997) indicate that the situation had changed little at the time of their survey and that librarians' knowledge of the literature was deficient, with five out of eight librarians surveyed unaware of key resources. 
These habits are perceived by the library profession as correctable by improved access (proposed solutions include in-ward libraries, bookclubs and so on) and by training which will improve the underdeveloped search skills of nurses (Capel et al., 1997). Cullum and her colleagues (Cullum et al., 1997), in an article promoting a new journal ('Evidence-based Nursing') published jointly by the British Medical Association and the Royal College of Nursing, observe that 'studies' show that research-based nursing care is more effective, though nurses do not inform their practice with research. They construe this as an access problem: relevant research is not always reported in nursing journals and nurses are not trained to 'find and appraise'.

Recent work on itinerant practitioners (midwives, health visitors) has looked at WWW as a way of overcoming physical access problems (Farmer \& Richardson, 1997). For the QMC group, travel from remote workplaces to access points (nearest university, nursing, or public library) makes physical remoteness an issue. The scope of what could be accessed is also problematic, as resources are scattered across different specialist libraries -- a point confirmed in the literature (Yeoh, 1995). In addition, the QMC group described navigation and searching difficulties: trained by library staff, they were only aware of simple keyword searches and found the process of iterative sifting that followed badly constructed strategies too time-consuming for them to rank the biomedical apparatus highly as a resource.

Even where they understand how the world of scientific evidence is constructed and is to be navigated, there is a deeper issue: a suspicion of the medical evidence that they are required to access, which emerges from the particular model of patient care on which their professional training is based (Hicks \& Hennessy, 1995; White, 1997). The accommodation of detail is a significant problem area: the randomized clinical trial, which is the most highly ranked standard for evidence-based medicine, has been designed to occlude detail in the interests of generalizable outcomes, and the nursing models currently presented to students in UK Higher Education institutions are heavily qualitative. If many of those who write for publication are garrulous when reporting on patients, they appear reticent when accounting for the interventions that constitute practice. Cutliffe (1997), in a discussion of nursing development units to foster best practice observes that experienced practitioners are often 'unable to put into words why they have behaved in a particular way' (p. 329). This point is developed further by Berragan (1998), who describes the difficulties often faced by practising nurses 'in defining what they do and hence what it is that constitutes nursing practice' (p. 211). She is sympathetic to this predicament, claiming that 'tradition and folklore still abound in nursing practice and are powerful sources of knowledge embedded in nursing culture' (p. 212).

\section{Domains in conflict}

As explained above, a migration to the university sector has been a major driver of 'professionalization' of nursing in the UK. An important part of this process, as of any professionalization program, is the establishment of a distinct body of knowledge. In the case of nurses, this involves a conscious dissociation of their practice from that of the medical domain. In the early years of separatism, models of the nursing process focused on distinct sets of tasks which defined the work of the nurse (McFarlane, 1970). More recently, an 'ethic of care' has been proposed as the distinguishing feature of the domain (Condon, 1992; White, 1997). Cutliffe 
(op.cit.) describes this as 'a particular philosophy or set of attitudes, a philosophy which sees them (nurses) as caring, committed, self-aware, individuals who view their clients in a holistic manner and seek to empower them' (p. 328). He draws on Benner's work (Benner, 1982) to posit power sharing within the therapeutic relationship as the objective of care - expert interventions, suffused with 'warmth and unconditional positive regard' (Cutliffe, op. cit. p. 330) will induce hope, a sense of control, and an improved quality of life. The 'new nursing professional' (Luker, 1997) focuses on nurse-patient empathy as much as technique or medical knowledge: 'the psychological and subjective nature of the body' is the focus, and narrative and subjective testimony are accepted as sources of knowledge. My QMC participants, like many other university-trained nurses in the UK, use the Roper model (Roper et al., 1980) to structure clinical practice in the ward. This focuses on lifestyle and lifecycle analysis to provide a holistic profile of the patient, which will extend the frame of reference of therapy beyond the immediate locus of clinical intervention.

Concomitant with this approach to practice, is a research bias towards methodologies (largely phenomenological) that embrace affect and intuition as primary dimensions of therapy. Hicks \& Hennessy (1997), discussing the EBM mandate, comment that 'emphasis on RCTs with relative marginalization of alternative, more qualitative forms of research, may seriously limit the nursing research database because of its inappropriateness for many nursing interventions' (p. 595) -- this point is also made by McCloskey \& Bulechek (1995) in relation to the Nursing Interventions Classification in the US. They suggest that the problem is structural - qualitative research is not favoured at either the funding or dissemination stages of the construction of the medical knowledge base in the UK; as a result, there is a lack of confidence among nursing professionals that they can conduct and evaluate research, and a lack of management commitment to nursing research. They claim that over-emphasis of RCT-based research has been counterproductive: in spite of heavy promotion, many in the nursing profession choose to ignore the texts - there is 'an entrenched disinclination...towards any role responsibilities which are not firmly embedded in direct caring activities' (p. 596). Positing 'mutual regard and respect, intuition and caritas' (p. 598) as the value system that underlies nursing practice, Hicks \& Hennessy describe the RCT as 'a methodology that conflicts with the nursing tradition, relegating it to an inferior position which by implication, has neither the right nor the might to make any real difference to clinical practice' (p. 599).

Taking a less strident line, Kitson (1997) suggests that a key criterion of professional intervention whether initiated by a doctor, nurse, or physiotherapist is 'does it do what it is intended to do?', and that this can only be judged on the basis of evidence based on clinical experience, or, at best, research evidence. . There is a difference between weighing an intervention against the scientific evidence and taking a critical approach to work and evaluating expected patient outcomes against implicit standards based on clinical experience and judgment; but, if a discipline is 'young', the former may not be easy to do. She reviews the literature on effective intervention and finds little to support the claims that individual relationships are critical to positive outcome, though educating patients is. Kitson questions the dominance of disease classification categories based on RCTs of pharmacological interventions and makes a plea for the inclusion of alternative approaches to diagnosis, which recognize the complementary tradition of 'clinical experience'. This, says Benoliel (1996), should not be interpreted in too narrow. While sympathetic to qualitative research, specifically the grounded theory approach, she states that 
there is 'a need within nursing to broaden current definitions of nursing practice beyond the narrow confines of the nurse-patient relationship as a one-to-one entreprise...[since] in the realworld of healthcare delivery, caregiving is offered by multiple nurses, not one...[T]he larger environment of the work setting influences how nurses practice as individuals, how they work as colleagues, and what happens to patients and families' ( $\mathrm{p}$ 418). Though there is a case for knowledge acquired by qualitative means to be included in the canon of practice, much of this work has not been rigorous or particularly persuasive (Baker et al., 1992) in ensuring that nursing knowledge per se is recognized as a powerful professional corpus, and there is dissent in the nursing profession as to how to strengthen the position of 'nursing knowledge' as a clinical warrant. Walsh (1997), for example, dismisses the 'empathic turn' and states that accountability in law is a key criterion of professional practice: 'Appeals to nebulous concepts of intuition and knowing in the doing will not impress the NHS trust or GP conscious of litigation and risk management. The nurse must be able to justify his or her actions with reference to an objective evidence base if he or she is to earn the authority that will make for truly accountable practice' (p. 41).

\section{Aligning domains: boundary objects and translation work}

The text so far has outlined a scenario where two domains, one well established (medical) and one newly professionalized (nursing), may be in conflict. Though both share clinical practice as an arena, and wish to attain common outcomes within that arena, there appears to be little epistemological and ontological common ground: the two domains are in a state of non-alignment. External directives, however, require them to overcome their differences in the interests of accountable service (National Health Service Executive, 1996). Since nursing, as a newly professionalized domain, is unlikely to abandon the traditions (in ethics, research, and practice) on which it bases its claim to separate status, some means of accommodating difference must be found. In the sections which follow, the role of the clinical practice guideline as a means of accommodation is explored.

There is, of course, a vocabulary and a theoretical framework to hand. In 1989, Star and Greisemer, in a discussion of the balance of heterogeneity and cooperation in organizations, proposed a class of objects which can accommodate these divergent trends (Star and Greisemer, 1989). These 'boundary objects' provide common ground for heterogeneous social actors to work together. They may be artefacts, texts, prescriptions, classification systems: they 'inhabit several intersecting social worlds' (p. 393), and are to some extent protean - 'plastic enough to adapt to local needs, and the constraints of the several parties employing them, yet robust enough to maintain common identity across sites' (p. 393). As they 'inhabit several intersecting social worlds and satisfy the informational requirements of each of them' (p. 393), boundary objects are essential components of information infrastructure. The creation and maintenance of boundary objects is a political process. Organizational actors who engage with boundary objects will seek to privilege their own concerns, and enlist allies with this end in view. In the museum case study where Star and Greisemer situate their discussion, boundary objects were a way of 'translating the concerns of the non-scientist into those of the scientist' (p. 404). 
In using 'translation' in this way, they draw on the practice of Latour and his colleagues. Translation happens through a process of 'inscription'; artefacts embody the interests of those who engage with them, and carry, as Star and Greisemer indicate, 'at every stage the traces of multiple viewpoints, translations and incomplete battles' (p. 413). Bowker, Timmermans and Star (1995) note that, from Latour's perspective, classification, a political resource, is central to the relationship between inscriptions, work practice and standards (p. 381). This corroborates Suchman's view (Suchman, 1994) that categorization devices are sites where internal and extrnal claims to organizational territory are contested. A further definition is offered here: an inscription, as a categorizing device, may be seen as a boundary object with attitude, a point developed further below.

Callon discusses inscription in terms of anticipated use (Callon, 1991). He sugests that we should ask four questions: what is being inscribed? who inscribes? how are inscriptions inscribed? and how powerful are the inscriptions?. By 'powerful', he invokes the scope and strength of the alliances that are embodied in a given inscription. Monteiro and Hansett (1995) discuss the 'power' of inscription in some detail in a study of EDI in the Norwegian health service, which draws heavily on Latour's Actor Network Theory [ANT] as an explanatory framework. ANT, says Monteiro, 'supports an inquiry which traces the social process of negotiating, redefining, and appropriating interests back and forth between an articulate, explicit form where they are inscribed within a technical artefact'. The larger the network within which inscriptions operate, the stronger the inscription; but a broad network may also dilute the power of inscription, as use becomes indeterminate: the boundaries of a boundary object are always in play. As we shall see below, UK clinical guidelines may be seen as both weak and strong inscriptions: there are many local variants, published under a number of different auspices, which makes it hard to talk in terms of a political centre; but critical bonds (the link between funding and NHS endorsed guidelines; for example) confer immense political clout, so that NHSsponsored guidelines are strong inscriptions indeed.

Bowker and Star and their colleagues have analyzed a number of classification systems, drawing on, inter alia, the boundary object framework. Points in common between their analysis and what may be observed in the development of clinical guidelines in the UK suggest that clinical guidelines may also be described as classification objects. As indicated above, a case that is pertinent to the development of clinical guidelines, is that of the Nursing Interventions Classification (Bowker, Timmermans \& Star, 1995), discussed in an ongoing exploration of 'the quiet politics of voice and values in information infrastructure' (p. 345). The NIC inscribes a 'vision of what nursing is and should be', and it coordinates 'bodies impairments, charts, reimbursement systems, vocabularies, patients and health care professionals' (p. 349). It has contributed to the strengthening of the political position of the nursing profession, as it has been included, in a number of major domain resources, including UMLS, 'a major victory for the development group'. The effectiveness of the NIC (or, as is claimed here, any classificatory inscription) may be judged, say Bowker and his colleagues, in terms of three dimensions: comparability (equivalence across sites which is based on 'regularity' in semantics and objects), visibility (or ability to accommodate what is 'wrongly invisible'), and control, or hospitality to appropriation by users (p. 347). These criteria are applied to guidelines in a later section of the text. 
In addition to the boundary work of Bowker, Star and their colleagues, work by Berkenkotter (1995) on 'boundary rhetoric' is relevant to claims being made here for clinical guidelines as translation artefacts. Berkenkotter, drawing on work by Fuller (1988) on interdisciplinary interpenetration, trading zones and boundary rhetoric, describes disciplines as 'contingent groupings of practitioners situated in multiple networks determined by such factors as objects of study, theories, methodologies, epistemological alliances, institutional sites and funding arrangements' (p. 177). Boundary rhetoric, says Berkenkotter, is primarily designed to accommodate disciplinary differences, though it may, over time, alter practice; it rallies support in a number of different fields 'through the creation of a heteroglossic text' (p. 179). 'Key informants', trusted authorities in a number of fields, can play an important role in enlisting and enrolling allies to support emerging rhetorics. The heteroglossic mix offered by the clinical guideline is potent: as indicated above, it embodies the filtering expertise of the searcher, the relevance judgments of clinicians, and the feasibility assessments of management staff in a practical guide to action.

\section{Clinical guidelines as translation artefacts}

The brief case study which follows presents an analysis of the development of clinical guidelines in Scotland and attempts to evaluate them as translation artefacts, boundary objects and inscriptions. Clinical guidelines are defined by the Scottish Intercollegiate Guideline Network (SIGN, 1998) as 'evidence-based national recommendations...that are translated by local practitioners into protocols which reflect particular local circumstances and styles of practice ... the available evidence is correctly synthesized and weighted within a guideline, so that, when followed, the guideline leads to improvements in health'. The SIGN secretariat is a network of clinicians and healthcare professionals that includes representatives of all the UK Royal Medical Colleges (the professional bodies which validate practitioners) as well as nursing, pharmacy, dentistry, and professions allied to medicine. Patients' views are represented on SIGN through the Scottish Association of Health Councils. SIGN works closely with other national groups and government agencies working in the National Health Service in Scotland.

The theoretical work of Bowker, Star, Timmermans, Berkenkotter, and Latour outlined above can throw light on the clinical guidelines developed in Scotland as 1) translations, 2) boundary objects and 3) inscription devices. In the first part of the paper, the intractability of the medical corpus as a source of clinical practice or 'care' was discussed in terms of three problem areas: structural efficiency, epistemological dissonance and ethical/ontological incommensurability. Structural inefficiency was described in terms of the literary apparatus and the different translations or surrogates that are produced to allow access to the archive: abstracts, indexes, codes and so on. Clinical guidelines effect yet one more transformation and solve the problems of structural efficiency by delegating searches to a cadre of motivated clinical domain specialists. This adds to the number of removes at which practice draws on literary warrant, but facilitates practice that fulfils Price's (1981) desideratum of turning knowledge into action on the basis of validation and evaluation 'by its affected community' (p. 8). The translation process which underlies clinical guidelines has, in the UK at least, been relatively transparent (Eccles et al., 1996): details are provided in a number of sources of the sources and search strategies involved. By classifying the literature in terms of recommendations for action, in a process endorsed by 
groups of named stakeholders who constitute the development team in any given case, the guideline cuts several epistemological knots.

The diffusion of guidelines is currently rather arbitrary. As indicated above, they are indexed inconsistently in the major databases: EMBASE thinks that any articles dealing with good clinical practice and clinical protocol may be indexed as types of practice guidelines. MEDLINE makes a clear distinction between clinical protocol and practice guidelines with no hierarchical relation between them, though they have a document type field that could be used. UK health librarians, not surprisingly, are unsure where to look when clients ask for guideline information; guidelines are, however, well represented on the Web (Kiley, 1998). A commercially published handbook (set up in June 1997) is sent three times yearly to GPs and other appropriate medical personnel free of charge: the aim 'is to provide those involved in developing and implementing primary care practice or local guidelines with a valuable reference source in a convenient, easy to use, working handbook' ('Guidelines', 1997, p. 2). The editors stress that it is not endorsed by the NHS, or the Clinical Outcomes Group [COG], and indicate that only COG-recommended RCTs should be used in contract specification. On balance, clinical guidelines appear to address some of the structural inefficiency problems posed by the traditional medical corpus.

In its role as a broker between dissonant ontological standpoints, the guideline can be seen to function as a boundary object. Measured in terms of Bowker's triad, it offers comparability: the recommendations that all local developers work on the basis of national and regional guidelines (as that is the level where expertise is likely to be available to carry out systematic reviews) is likely to be heeded, and the guidelines which have the backing of the NHS or the professional bodies (as is the case of SIGN) will have larger reach, as they have greater validating power in funding allocations. In terms of enhancing visibility, the ranking system allows guidelines to be hospitable to non RCT-based, non drug-driven treatment description, and though such descriptions may not be highly ranked, the fact that they are listed is evidence of the inclusiveness of the project. The SIGN report on Epilepsy, for example, includes 'numerous examples of patient leaflets and booklets' gathered by the clinical nurse specialist with help from the Epilepsy Association for Scotland, ranked as Grade C material. ('Grade $C^{\prime}$ indicates either absence of directly applicable clinical studies of good quality or an extrapolation of level 1,2 or 3 evidence). Weak warrant aside, the role of the clinical nurse is foregrounded in this guideline: she 'would act as educator and trainer for staff and patients, audit co-ordinator, and facilitator in the implementation of local protocols'. (SIGN, 1997, p. 26)

In terms of control, or openness to user adaptation, Bowker's third criterion, the UK clinical guidelines score highly. SIGN' participative philosophy is typical of groups that develop clinical guidelines:

'It is expected that this guideline will be adopted after local discussion involving clinical staff and provider and purchaser management. The Area Clinical Audit committee should be fully involved. Local arrangement will then be made for the derivation of specific local protocols to implement the national guideline in individual hospitals, units and practices and for securing compliance with them. This will be done by a variety of means including patient specific reminders, 
continuing education and training, and clinical audit. Service contracts will reflect the arrangements fully along with their related costs'.

Though local developers may deviate from national guidelines, they should document any significant departures, explain the reasons for the differences and document these in patients' notes.

As an inscription device, the clinical guideline instantiates the political dynamic of the stakeholders whom it engages. If inscriptions are, as suggested above, boundary objects with attitude, 'attitude' in the case of the clinical guideline is achieved in the value judgments implied by the ranking mechanism. Assessed in terms of Callon's four questions (what?, who?, how? and how powerful?), the clinical guideline can be seen as an effective fulcrum (Fuller, 1994). In terms of aligning the non-aligned, the main purpose of inscription according to Callon, the major work has been done behind the scenes by government, the invisible hand, which has made the RCT the ultimate standard for care and indicated that only RCT evidence should be invoked in contracts for the purchase of health care: what is being inscribed is the medical point of view, though, in a very British compromise, however, other 'ways of knowing' are invited to the party, though not to sit at the High Table. This can be compared with the situation in France (Durand-Zaleski et al., 1997), where doctors are fined for non-compliance with RCT based guidelines. Those at the High Table (who 'do' the inscription) are the medical profession, allied in different ways according to the therapeutic focus of the guidelines, with other practitioners. A crude measure of the balance of power within a guidelines is the membership list for each development team: medical staff are predominant. The 'how' of the inscription is its most compelling dimension: as suggested above, the guideline offers a powerful heteroglossic mix, which can both solve problems and consolidate points of view.

\section{Conclusion}

The paper has reviewed problems that arise when texts are to be translated into healthcare: unwieldy literature structures, difficulties in access and epistemological and ontological dissonance, It has explored the role and status of the clinical guideline in overcoming inhibitors to evidence-based healthcare. Clinical guidelines are an interesting hybrid, combining retrieval (with peer assessment) and prescriptive classification (a merging of clinical with organizational assessment) in a novel mix of literary, clinical and administrative warrants. Their power (Callon's fourth critical dimension) as inscriptions is yet to be determined, as guideline development in the UK is in its early stages. Guidelines secretariats can amplify the power of inscription by activating networks of strong and weak ties. This may be done in a number of ways: SIGN recommends certain weblinks to readers of its web page (weak ties); it also works closely with other local agencies, like the Scottish Health Purchasing Information Group (strong tie), who have recently proposed the joint development of a 'Guideline for Leg Ulcer Treatment': 'There is a dearth of comparative studies to inform choice between treatments compared with the number of placebo-controlled studies which show a wide range of treatments to be effective...[We propose] a study to see the effect on leg ulcer care of implementing a national [SIGN] guideline with the aid of an education programme for community nurses' (p. 4). (SPHIC, 1996) 
Proceedings of the 9th ASIS SIG/CR Classification Research Workshop

Sceptics may claim that unless guidelines are tied specifically to audit, nursing practitioners will continue to work on the basis of the knowledge base that is familiar to them. Optimists (or those who may wish to engage strategically with the inscription process) visualize a forum to present a nursing point of view, sharpened by improved quality of research. Attempts to have qualitative nursing research included in the DARE database (one of the sources of systematic review) might be interpreted as a move of this sort (Boynton, 1995). Externally, the inscriptive power of guidelines can be improved by inclusion in major indexes. Their hybrid power to link warrant and activity and provide evidence of 'turning knowledge directly into action' (p. 8), thus closing 'Price's loop', might make them a useful addition to ISI's portfolio, for example: guideline citations may serve as value-added currency in the cognitive economy.

\section{Acknowledgements}

The author wishes to thank Martin Higgins for help with sources, Justus Krabshuis for his 'key informant' advice, Herbert Snyder for comments on the first draft of this paper, and Elin Jacob for further comments. Thanks are also due to members of the Modular Masters Research Methods course at Queen Margaret College, particularly to Nicole, Susan, Joanne and Erica.

\section{References}

Baker, C., Wuest, S., \& Stern, P.N. (1992). Method slurring: the grounded theory/ phenomenology example. Joumal of Advanced Nursing, 17, 1355-1360.

Bawden, D. \& Robinson, K. (1997). Information behaviour in nursing specialities: a case study of midwifery. Journal of Information Science , 23(6), 407-421.

Benner, P. (1982). From novice to expert. American Journal of Nursing, 402-407.

Benoliel, J.Q. (1996). Grounded theory and nursing knowledge. Qualitative Health Research, $6(3), 406-428$.

Berkenkotter, C. (1995). Theoretical issues surrounding interdisciplinary interpenetration. Social Epistemology, 9(2), 175-187.

Berragan, L. (1998). Nursing practice draws upon several different ways of knowing. Journal of Clinical Nursing, 7, 209-217.

Bowker, G., Timmermans, S. \& Star, S.L. (1995) "Infrastructure and organizational transformation: classifying nurses' work". In W.Orlikowski, G.Walsham, M.R.Jones, \& J.I. DeGross (Eds.), Information technology and changes in organizational work. Proceedings of the IFIP WG8.2 working conference on information technology and changes in organizational work, December 1995. (pp. 344-370). London: Chapman and Hall. 
Bowker, G. \& Star, L. (1997). "Science, accounting and administrtion: the worlds of the ursing intervention classification". Paper presented at the ASIS SIGCR workshop, "Classificatory structures and the construction of reality". In C. Schwarz and M. Rorvig (Eds.), Proceedings of the 60th ASIS Annual Meeting November 1-6, Washington, 1997. Medford, NJ: ASIS/Information Today, 376.

Boynton, J., Glanville, J., McDaid, D., \& Lefebvre, C. (1998). Identifying systematic reviews in MEDLINE: developing an objective approach to search strategy design. Journal of Information Science, 24(3), 137-157.

Callon, M. (1991). "Techno-economic networks and irreversibility". In J. Law (Ed). A Sociology of monsters: essays on power, technology and domination (pp. 132-161). London: Routledge.

Capel, S., Banwell,L., \& Walton, G. (1997). Library and information services to support the education and development of nurses: the management of cooperation and change - a clash of two cultures? Health Libraries Review, 14 (4), 233-245.

Cimino, J. (1995). Vocabulary and healthcare information technology: state of the art. Journal of the American Society for Information Science, 46(10), 777-782.

Cochrane, A. L. (1972). Effectiveness and efficiency: random thoughts in health services. London: Nuffield Provincial Hospital Trust.

Condon, E.H. (1992). Nursing and the caring metaphor: gender and political influences on an ethics of care. Nursing Outlook, 40(1), 14-19.

Cullum, N., DiCenso, A., \& Ciliska, D. (1997). Evidence-based nursing: an introduction. Nursing Standard, 11(28), 32-33.

Cutliff, J. R. (1997) The nature of expert psychiatric nurse practice: a grounded thoery study. Journal of Clinical Nursing, 6, 325-332.

Department of Health. (1991). Research for health. A research and development strategy for the NHS. HMSO: London.

Durand-Zaleski, I., Colin, C., \& Blum-Boisgard, C. (1997). An attempt to save money by using mandatory practice guidelines in France. $B M J, 7113,11$ October.

Eccles, M., Clapp, Z., Grimshaw, J., Adams, P., Higgins, H., Purves, I., \& Russell, I. (1995). Developing valid guidelines: methodological and procedural issues from the North of England evidence based guideline development project. Quality in Health Care, 5, 44-56.

Editorial.Guidelines, Volume 3. October 1997. 
Proceedings of the 9th ASIS SIG/CR Classification Research Workshop

Farmer, J. \& Richardson, A. (1997). Information for trained nurses in remote areas: do electronically networked resources provide an answer? Health Libraries Review, 14, 97-103.

Fuller, S. (1988). Social Epistemology. Bloomington, IN: Indiana University Press.

Fuller, S. (1993) Philosophy, rhetoric and the end of knowledge: the coming of science and technology studies. Madison: University of Wisconsin Press.

Gerson, E.M \& Star, S.L. (1986). Analyzing due process in the workplace. ACM Transactions on Office Systems , 4(3), 257-270.

Henry, S.B. (1992). Nursing informatics: state of the science. Journal of Advanced Nursing, 22, $1182-1192$.

Hicks, C. \& Hennessy, D. (1995) Mixed messages in nursing research: their contribution to the persisting hiatus between evidence and practice. Journal of Advanced Nursing, 25, 595-601.

Jacob, E. K., \& Albrechtsen, H. (1997). Constructing reality: the role of dialogue in the development of classificatory structures. In Proceedings of the 6th International Study Conference on Classification Research.

Kiley, R. (1998). Evidence-based medicine on the Internet. Journal of the Royal Society of Medicine, Volume 91, 74-75.

Kitson, A. (1997). Using evidence to demonstrate the value of nursing. Nursing Standard, $11(28), 34-39$.

Kling, R. (1987). "Defining the boundaries of computing across complex organizations". In R.J. Boland and R.A. Hirscheim (Eds.), Critical Issues in Information Systems Research, (pp. 307-362). Chichester: John Wiley and Sons.

Krabshuis, J. (1997). Endoscopy information online: can endoscopists close the gap between what is known and what they do? Endoscopy, 29, 871-892

Krabshuis, J. (1998). Personal communication, June 10.

Latour, B. (1991). "Technology is society made durable". In J. Law (Ed), A Sociology of monsters: essays on power, tehchology and domination, (pp. 103-131). London: Routledge.

Luker, K. (1997) Research and the configuration of nursing services. Journal of Clinical Nursing, 6, 259-267.

McCloskey, J. C. and Bulechek, L.M. Nursing Interventions Classification (NIC): development and use. In R.P. Schwarz et al. (Eds.) Proceedings of the 6th ASIS/SIG/CR Classification Research Workshop. ASIS/SIG/CR, 1995. 111-132. 
Davenport, E. (1998). Translating texts into care: Classification issues raised by evidence-based practice in the UK health sector. 9th ASIS SIG/CR Classification Research Workshop, 53-70. doi:10.7152/acro.v9i1.12744

Proceedings of the 9th ASIS SIG/CR Classification Research Workshop

McFarlane, J. (1970). The proper study of the nurse. London: Royal College of Nursing. 
McKibbon, K.A., Wilczynski, N., Hayward, R.S., Walker-Dilks, C.J. and Haynes, B. (1995) The medical literature as a resource for health care practice. Journal of the American Society for Information Science, 46(10), 737-742.

Meadows, J. (1987). The origins of information science. London: Taylor Graham.

Monteiro, E. \& Hansett, O. "Social shaping of information infrastructure: on being specific about the technology". In In W.Orlikowski, G.Walsham, M.R.Jones, \& J.I. DeGross (Eds.), Information technology and changes in organizational work. Proceedings of the IFIP WG8.2 working conference on information technology and changes in organizational work, December 1995. (pp. 325-343). London: Chapman and Hall.

NHS Executive (1996). Promoting clinical effectiveness: a framework for action in and through the NHS. London: Department of Health.

NHS. (1996). Clinical guidelines. Using guidelines to improve patient care within the NHS. July 1996. London: Department of Health.

Price, D. de Solla. (1981). "The development and structure of the biomedical literature". In K. S. Warren (Ed.), Coping with the biomedical literature: a primer for the scientist and the clinician (pp. 3-16). New York: Praeger.

Rector, A.L., Glowinski, A.J., Nowlan, W.A. \& Rossi-Mori, A. (1995) Medical concept models and medical records: an approach based on GALEN and PEN\&PAD. Journal of the American Medical Informatics Association, 2(1), 19-35.

Roper, N., Logan,W. \& Tierney, A. (1980) The elements of nursing. Edinburgh: Churchill Livingstone.

Royal College of Nursing. (1996). Clinical effectiveness and evidence based practice. Royal College of Nursing, London.

Royal College of Nursing (1996). The Royal College of Nursing Clinical Effectiveness Initiative - $a$ strategic framework. London: Royal College of Nursing.

SHPIC. (1996). Scottish Health Purchasing Information Centre. Leg ulcers. Summary. http://www.nhsconfed.net/shpic/doc06.htm

SIGN. (1998). Scottish Intercollegiate Guidelines Network. http ://pc47. cee. hw.ac.uk /sign /intro.htm

SIGN. (1997). Diagnosis and management of epilepsy in adults. A national clinical guideline recommended for use in Scotland by the Scottish Intercollegiate Guidelines Network. Pilot edition, November 1997. Edinburgh: SIGN. 
Star, S.L. \& Griesemer, J.R. (1989). Institutional ecology,'Translations' and boundary objects: amateurs and professionals in Berkeley's Museum of Vertebrate Zoology, 1907-1939. Social Studies of Science, 19, 387-420.

Star, S.L. Slouching towards infrastructure. http://www.gslis.ucla.edu/DL/star.html

Suchman, L. (1994). Do categories have politics? The language/action perspective reconsidered. Computer Supported Cooperative Work, 2, 177-190.

Urquhart, C. \& Crane, S. Nurses' information-seeking skills and perceptions of information sources: assessment using vignettes. Journal of Information Science, 20(4), 237-246.

Urquhart, C. and Crane, S. (1995) Preparing for post-registration education and practice (PREP): the support role of the library. Nurse Education Today, 15, 459-464.

Walsh, M. (1997) Accountability and intuition: justifying nursing practice. Nursing Standard, 11 (23) $39-41$

White, S.J. (1997) Empathy: a literature review and concept analysis. Journal of Clinical Nursing, 6, 253-257.

Williams, J. (1996) Do we need a classification for primary care? Journal of Information Research in Primary Care, March, 11-13.

Yeoh, J. (1995). "Nursing, midwifery and health visiting". In M. Carmel (Ed.), Health care librarianship and information work (pp.20-31). Second edition. London: Library Association. 
Davenport, E. (1998). Translating texts into care: Classification issues raised by evidence-based practice in the UK health sector. 9th ASIS SIG/CR Classification Research Workshop, 53-70. doi:10.7152/acro.v9i1.12744

Proceedings of the 9th ASIS SIG/CR Classification Research Workshop 